\title{
Le grand saut des organisations de producteurs agricoles africaines
}

De la protection sous tutelle à la mondialisation

\section{A giant step for African agricultural producers' organizations} From regulated protection to globalization

\section{Pierre-Marie Bosc, Jacques Berthomé, Bruno Losch et Marie-Rose Mercoiret}

Numéro 285, juillet 2002

Les entretiens de Maraussan. Qualité, équité : les stratégies coopératives

Maraussan Special Issue. Quality and Equity: Cooperative Strategies

URI : https://id.erudit.org/iderudit/1022251ar

DOI : https://doi.org/10.7202/1022251ar

Aller au sommaire du numéro

\section{Éditeur(s)}

Institut de l'économie sociale (IES)

ISSN

1626-1682 (imprimé)

2261-2599 (numérique)

Découvrir la revue

Citer cet article

Bosc, P.-M., Berthomé, J., Losch, B. \& Mercoiret, M.-R. (2002). Le grand saut des organisations de producteurs agricoles africaines : de la protection sous tutelle à la mondialisation. Revue internationale de l'économie sociale, (285), 47-62. https://doi.org/10.7202/1022251ar
Résumé de l'article

Dans le contexte de développement étatique qu'a connu l'Afrique subsaharienne des indépendances aux années 80 , l'émergence du mouvement coopératif s'est réalisée sous contrôle de l'administration et les fonctions économiques officiellement reconnues et " dévolues " aux organisations de producteurs étaient strictement limitées. Parallèlement, les organisations locales prenaient en charge des actions sociales en s'efforçant de pallier la défaillance des pouvoirs publics. Avec la libéralisation économique et politique et le désengagement des États, les organisations de producteurs ont été confrontées brutalement à un nouvel environnement beaucoup plus concurrentiel et instable. Elles doivent cependant relever le défi du transfert de nombreuses fonctions économiques et faire face, dans le même temps, à des demandes sociales croissantes liées à la dégradation de nombreuses situations nationales. Les formes d'organisation de la période actuelle ne font pas référence à un modèle prédéfini, mais dépendent essentiellement des interactions entre les dynamiques propres aux organisations et les changements à l'oeuvre dans leur environnement économique, institutionnel et politique. 


\section{LE GRAND SAUT DES ORGANISATIONS DE PRODUCTEURS AGRICOLES AFRICAINES}

De la protection sous tutelle à la mondialisation

(*) Cirad-Tera, progranme « Agri cultures familiales »: TA 60/15, 73, avenue Jean-François-Breton, 34398 Montpellier cedex 5.

UMR Moisa : 2, place Viala. 34060 Montpellier cedex 2.

(**) Ciepac: 930, chemin des Man drous, 34170 Castelnau-le-Lez.

$(* * *)$ Cirad-Tera, programme « Agricultures familiales 》: TA 60/15, 73, avenue Jean-François-Breton, 34398 Montpellier cedex 5. par Pierre-Marie Bosc ${ }^{(*)}$, Jacques Berthomé ${ }^{(*)}$, Bruno Losch ${ }^{(*)}$ et Marie-Rose Mercoiret ${ }^{(* * *)}$

Dans le contexte de développement étatique qu'a connu l'Afrique subsaharienne des indépendances aux années 80, l'émergence du mouvement coopératif s'est réalisée sous contrôle de l'administration et les fonctions économiques officiellement reconnues et " dévolues " aux organisations de producteurs étaient strictement limitées. Parallèlement, les organisations locales prenaient en charge des actions sociales en s'efforçant de pallier la défaillance des pouvoirs publics. Avec la libéralisation économique et politique et le désengagement des Etats, les organisations de producteurs ont été confrontées brutalement à un nouvel environnement beaucoup plus concurrentiel et instable. Elles doivent cependant relever le défi du transfert de nombreuses fonctions économiques et faire face, dans le même temps, à des demandes sociales croissantes liées à la dégradation de nombreuses situations nationales. Les formes d'organisation de la période actuelle ne font pas référence à un modèle prédéfini, mais dépendent essentiellement des interactions entre les dynamiques propres aux organisations et les changements à l'auvre dans leur environnement économique, institutionnel et politique.

es économies à l'échelle de la planète sont confrontées depuis mainT tenant plus de vingt ans à un processus de libéralisation dont l'extension et la généralisation constituent l'un des principaux attributs de la mondialisation. Celle-ci fait suite au "temps du développement" (Mc Michael, 1996), qui a suivi la Seconde Guerre mondiale et a accompagné le processus de décolonisation, où le rattrapage des Etats-nations " sous-développés " correspondait à une vision du monde et à un objectif incontestés.

Les institutions de Bretton-Woods (FMI et Banque mondiale), alors créées pour promouvoir les coopérations économique et financière internationales, ont bien sûr adhéré à cette perspective et mis en œuvre des interventions où le développement était clairement envisagé dans un cadre national. D'inspiration keynésienne, avec la reconnaissance du rôle de l'Etat dans la correction des défaillances de marché et dans l'impulsion 
des dynamiques économiques, leur action a connu un tournant radical à compter des années 80 . Le processus de libéralisation engagé en réponse à la crise du mode de croissance de l'économie mondiale (avec en particulier la recherche de nouveaux débouchés pour les firmes des pays industrialisés) s'est traduit par l'adoption de nouvelles conceptions du développement et de la stabilisation économique (Stiglitz, 2002). Ce nouveau consensus (souvent dénommé " de Washington ») s'est traduit par un changement radical des politiques, le plus souvent imposé par les agences internationales, où les anciennes régulations et protections publiques étaient démantelées au profit d'une ouverture des économies à travers la réduction des barrières à l'entrée, le désengagement des Etats de la production de biens et services et les privatisations.

Pour les organisations de producteurs agricoles africaines, qui avaient connu un développement encadré par les Etats à compter des indépendances au tournant des années 60 , ce passage du cadre national à la mondialisation correspond à un grand saut dans un environnement méconnu et non mầtrisé, caractérisé par une croissance des instabilités, de la concurrence et des asymétries. La confrontation des différents modèles agricoles sur les marchés internationaux, notamment avec la mise en œuvre de l'accord agricole du cycle de l'Uruguay en 1994 et la création de l'Organisation mondiale du commerce, a mis en évidence des inégalités croissantes entre des agricultures caractérisées par de profondes disparités en termes de niveaux de productivité, de soutiens publics et de poids relatif au sein de leurs économies et de leurs sociétés. Il est possible de distinguer trois grands types de situations:

- d'un côté, les pays qui, de par leur position économique dominante, ont les moyens politiques et économiques de résister au processus de libéralisation des marchés agricoles, en préservant des niveaux élevés de soutien à la production et à l'exportation ainsi que de fortes protections intérieures (cas du Japon, de l'Union européenne et des Etats-Unis) ;

- à l'opposé, les pays qui, de par leurs dotations en facteurs et avantages comparatifs, ont joué le jeu de la compétitivité économique et de la libéralisation qui leur permettait de gagner des parts sur les marchés mondiaux libéralisés (cas des pays du groupe de Cairns) ;

- enfin, et c'est le cas de la très grande majorité des pays des Suds, les pays qui ont été contraints par l'étau de la dette à s'engager dans la réduction drastique de l'intervention de l'Etat, puis à libéraliser les échanges agricoles, souvent au détriment de productions nationales moins compétitives.

Ces inégalités entre types d'agricultures s’accompagnent de déséquilibres croissants entre les acteurs de l'agriculture eux-mêmes, dans chaque situation locale. En effet, dans bien des cas, les producteurs et leurs organisations se retrouvent face à des firmes mondiales issues des recompositions économiques et du mouvement de privatisation (marqué par l'ampleur des opérations de fusions et acquisitions parmi les entreprises de la première et de la seconde transformation). Ces firmes, dont certaines " pèsent " plus que de nombreux Etats, sont toutes originaires des pays industrialisés. 
(1) Il convient de rappeler que ces soutiens sont restés globalement inchangés depuis les années 80 . Ils étaient estimés à 274 milliards de dollars en 1998 (Hervieu et al., 2000), au profit des $4 \%$ d'actifs agricoles des pays développés.
Elles sont particulièrement actives dans les marchés des produits tropicaux et c'est avec elles que les organisations professionnelles se doivent aujourd'hui de négocier.

La période actuelle est donc « sensible ». Ce grand saut des organisations de producteurs dans le nouveau rapport de force, particulièrement critique en Afrique du fait de l'ampleur des asymétries, correspond à un enjeu majeur: celui du devenir des agricultures familiales qui, dans les pays des Suds, occupent encore 1,3 milliard d'actifs agricoles (96\% des actifs agricoles mondiaux) et font vivre 2,5 milliards de personnes, soit $41 \%$ de la population mondiale (Campagne, 1998; Losch, 2002), mais qui doivent aussi faire face à la concurrence déloyale des soutiens colossaux accordés par les grands pays industrialisés ${ }^{(1)}$.

C'est donc en référence à ce contexte de rupture que nous examinerons la trajectoire suivie sur la période 1960-2000 par les organisations paysannes et rurales africaines, en centrant plus particulièrement notre analyse sur le contexte ouest-africain. Après un rappel des configurations initiales, nous présenterons les formes d'adaptation originales qui ont été mises en œuvre, puis l'état et les enjeux des configurations actuelles.

\section{Esquisse des trajectoires historiques des organisations paysannes et rurales africaines à partir des indépendances (1960-1980)}

A quelques rares exceptions, le continent africain a été colonisé et les Etatsnations actuels découlent d'une filiation directe avec cette période. Sans remonter aux racines historiques des formes collectives d'organisation proposées par les colonisations française ou britannique (Chauveau, 1994), l'analyse des trajectoires des organisations paysannes et rurales africaines ne peut se faire sans un retour sur les choix des Etats nouvellement indépendants, au tournant des années 60 . Ceux-ci restent marqués par une idéologie du développement, issue des politiques de "rattrapage " économique et social de la fin de la période coloniale, et par les clivages géopolitiques liés au contexte de la "guerre froide " (cf. Sarraut-Woods, 1998, pour une mise en perspective des évolutions et filiations institutionnelles).

\section{Un choix massif en faveur des formes coopératives dès les indépendances}

\section{Des points communs au-delà des orientations politiques et idéologiques}

Au moment des indépendances, l'organisation collective - plus ou moins poussée - semble être un moyen indispensable pour accompagner les dynamiques de développement agricole; on retrouve alors dans le discours le recours au collectif, quelle que soit l'orientation politique ou idéologique affichée, sinon suivie, par les nouveaux Etats-nations. Ces choix se traduisent par la mise en place de coopératives dans de nombreux pays. 
(2) Seuls quelques pays ont fait exception à ce mouvement géné ral, en particulier la côte d'Ivoire où l'alliance entre le pouvoir poli tique et les intérêts privés étran gers a structuré durablement l'économie politique locale (cf. Losch, 2000).
Elles étaient considérées, dans la version du " socialisme africain ", comme un des moyens d'intégrer, de réconcilier les solidarités dites " traditionnelles » et la modernisation agricole et de donner ainsi corps à une " troisième voie " revendiquée par certains pays (Sénégal, Niger, Tanzanie). Dans leur version marxisante et, à des degrés divers et pour des durées variables selon les pays, les coopératives ont été perçues à plusieurs reprises par certains dirigeants politiques comme un moyen d' "introduire le socialisme dans les campagnes ", notamment à travers diverses tentatives - généralement infructueuses - visant à passer de la petite production individuelle à l'exploitation collective avec l'objectif de mettre en œuvre la nécessaire révolution scientifique, technique et sociale.

Sur le plan économique, la rupture des indépendances s'est traduite bien souvent par la désorganisation du commerce en provoquant le départ - spontané ou forcé - des sociétés commerciales coloniales ${ }^{(2)}$. En conséquence, au-delà des références ou options idéologiques, et faute de capitalisme local et de structures commerciales privées endogènes, il fallut généralement en créer de toutes pièces en combinant fréquemment deux dispositifs: des offices d'Etat de commercialisation et des coopératives " primaires » chargées de la collecte et du groupage des produits agricoles.

\section{Des différences notables selon les contextes politiques et économiques}

Selon les contextes politiques et les choix de politique économique, il est toutefois possible d'observer un gradient dans les formes collectives d'organisation et dans les contraintes qu'elles ont entraînées pour les paysanneries. On peut ainsi distinguer des formes autoritaires comme l'Ujaama (Tanzanie), avec déplacement de population et des conséquences qui dépassent le strict cadre agricole, ou les fermes d'Etat (Fapa) en Guinée, qui constituent un échec agricole patent, mais aussi des formes coopératives évoluant dans un environnement politique et économique plus «libéral ", telles les coopératives d'approvisionnement, crédit et commercialisation au Sénégal ou au Niger. Des configurations «alibi » ont aussi été fréquemment mises en œuvre: c'est le cas en Côte d'Ivoire dans le secteur café cacao où un système mixte, associant une production et une commercialisation privées sous tutelle de l'Etat, s'est traduit par la création de "groupements à vocation coopérative " (GVC) qui seront cantonnés au seul stade de la vocation durant plusieurs décennies (cf. Losch, 2001)

\section{Des coopératives en dehors de la paysannerie}

Ces coopératives sont globalement restées " étrangères " à la paysannerie, à tel point que l'on a pu parler de " coopératives sans coopérateurs». Les formes d'organisations importées, directement inspirées des organisations coopératives nées dans les pays industrialisés au tournant du XX ${ }^{\mathrm{e}}$ siècle, se sont trouvées plaquées sur des réalités dont la propension à la " coopération » avait été largement surestimée sur la base d'analyses erronées exaltant les valeurs collectives des sociétés africaines (Augé, 1973). Très 
rarement impliqués au départ, et généralement exclus des processus de décision et de contrôle de l'appareil coopératif, les producteurs n'ont jamais eu leur place dans un mouvement fortement encadré. En fait, ces coopératives ont été mises en place par les fonctionnaires et les notables ruraux; elles ont également servi les intérêts des partis politiques en tant que source de financement et outil de propagande.

\section{Une diversification induite ou spontanée des formes d'organisation}

\section{Le temps des projets}

Durant la fin des années 60 , mais surtout pendant les années 70 et 80 , les Etats et les bailleurs de fonds développent des formes d'intervention caractérisées par la mise en œuvre de projets de type "filière " plus ou moins intégrés. Situés souvent à l'échelle régionale, autour d'une culture "motrice " souvent d'exportation (le coton) ou d'une innovation " transformatrice " (l'irrigation), les projets mobilisent un personnel et des moyens spécifiques pour atteindre des objectifs précis.

Prévus au départ pour des durées limitées, les projets verront fréquemment leurs objectifs initiaux s'élargir progressivement vers la mise en œuvre d'un développement intégré régional (cas de la CMDT au Mali-Sud dans les zones cotonnières) et leur durée de vie se prolongera souvent du fait de phases correspondant à autant de tranches de financement extérieur. L'intervention par le biais des projets contribue le plus souvent à un affaiblissement des formes traditionnelles de l'action publique mises en place par les Etats au moment des indépendances comme au Sénégal (Banque mondiale, 1989), car les moyens sont progressivement affectés majoritairement aux projets.

Ces interventions publiques s'appuient sur des organisations fonctionnelles définies pour relayer et démultiplier l'action des projets sur des points précis (Colin et Losch, 1990). Les formes d'organisation utilisées vont certes varier d'un pays à l'autre et donneront lieu dans certains pays à de multiples statuts qui permettront à ces organisations de fonctionner: les associations villageoises au Mali dans la zone cotonnière à partir de 1974, les groupements d'intérêt économique (GIE) au Sénégal dans le cadre de projets régionaux ou catégoriels (Casamance dans les années 1975-1990) ou des groupements qui s'identifient soit par le nom du projet qui en est l'origine, soit par le village (les groupements RC2 du nom du projet de relance de la caféiculture en Guinée à la fin des années 80, par exemple). Tous les grands projets ont promu ces formes d'organisation qui se sont construites le plus souvent en dehors du système coopératif pour échapper à certains de leurs dysfonctionnements, parfois à la demande des paysans eux-mêmes. Les associations et groupements des zones cotonnières sont nés avec les marchés autogérés qui assurent la commercialisation primaire du coton, d'abord dans la région de Mali-Sud, puis dans les autres pays, et leurs fonctions se sont progressivement étoffées. Dans les périmètres irrigués des zones sahéliennes, des organisations ont également été mises en 
place sur la base de fonctions économiques autour des filières riz, mais aussi pour contribuer à la gestion des périmètres irrigués (gestion de la redevance et de l'entretien des canaux).

Simultanément, certains Etats ont promu à travers leurs services techniques spécialisés des programmes nationaux destinés à des catégories sociales ou professionnelles particulières (jeunes, femmes, pêcheurs, artisans). Ces interventions se sont, elles aussi, accompagnées de la création d'organisations spécifiques.

Ainsi, au fil des années, les options successives de développement rural ont généré des organisations nombreuses et diverses qui coexistent, depuis le village jusqu'au niveau national, formant des strates plus ou moins vivaces ou sclérosées à partir desquelles pourrait s'effectuer une relecture des interventions de développement qui se sont succédé ou ont coexisté. La conception de l'organisation qui caractérise cette période est purement fonctionnaliste, définie le plus souvent de manière unilatérale par l'intervenant et circonscrite dans l'espace villageois, voire infra-villageois, dans le cas de groupements catégoriels ou professionnels spécifiques (groupements de jeunes, groupements féminins, groupements de pêcheurs).

\section{Des dynamiques alternatives d'organisation}

En marge des interventions officielles de l'Etat se développent des initiatives d'inspirations différentes qui trouvent leur origine dans les réactions aux changements climatiques, notamment dans les régions sahéliennes, dans le développement des organisations non gouvernementales d'appui et dans les réactions des paysans aux formes directives des interventions des périodes antérieures. Dans les années 70 et 80 , deux grands types d'organisations peuvent être distingués: les organisations liées à des interventions locales et les organisations liées au mouvement associatif.

\section{Les organisations liées à des interventions locales}

Créées avec l'appui des ONG, de certaines coopérations bilatérales ou internationales, en collaboration parfois avec les services techniques nationaux marginalisés par les grands projets, ces organisations très nombreuses revêtent des formes diverses. A côté d'organisations " communautaires " ayant des objectifs multisectoriels et se réclamant parfois de solidarités traditionnelles, on trouve des groupements plus spécialisés, centrés sur une production (le maraîchage), sur une fonction économique en amont ou en aval de la production (comités de gestion des banques de céréales, de caisses d'épargne et de crédit) ou orientés vers l'appui à une catégorie sociale (les femmes) ou professionnelle.

\section{Les organisations paysannes liées au mouvement associatif}

Ces organisations résultent d'une initiative locale et sont autonomes par rapport à l'Etat lorsqu' elles ne se sont pas construites en opposition ouverte avec les formes directives d'intervention qui étaient alors promues (cas du Cadef en Casamance). Historiquement, ces organisations 
locales au départ se sont surtout développées au Sénégal et au Burkina Faso, où elles ont pris la forme d'organisations paysannes intervillageoises avec des statuts divers d'associations, d'ONG ou même de GIE, parfois difficilement acquis. Le mouvement s'est développé dès le début des années 70 et parfois avant. Parmi les organisations pionnières, on peut citer l'Entente de Bamba Thialène, l'association des jeunes agriculteurs de Casamance, l'Amicale du Walo au Sénégal et les groupements Nam au Burkina Faso.

Ce qui caractérise le mouvement associatif qui émerge à cette époque, c'est le rejet des formes dirigistes ou non concertées que revêt l'intervention publique, le constat du peu de résultats de ces interventions et la prise en charge par les acteurs eux-mêmes de la recherche de solutions à leurs problèmes, notamment dans des zones marginales du point de vue du développement des grandes filières et de leur potentiel agroéconomique. C'est aussi la prise de parole de certaines catégories sociales qui vont investir massivement ces nouvelles structures.

Les organisations - quelle que soit leur origine - se trouvent diversement renforcées par les transferts de responsabilités et de charges qui tendent à s'accroître au profit des organisations paysannes et rurales durant les années 90 (Mercoiret et al., 1997).

\section{Conclusion partielle: une chronique de faillites annoncées}

Tenter de dresser un bilan des expériences coopératives africaines n'est guère encourageant; mais ces analyses se doivent de tenir compte des contextes économiques et politiques dans lesquels ces formes d'organisation ont été promues. Certaines de leurs fonctions économiques ont été appréciées par les producteurs lorsque le système disposait de financements publics (accès à la mécanisation, commercialisation "plus honnête ", accès au crédit - souvent mal remboursé -, infrastructures...) et que ces actions palliaient le départ des structures privées coloniales qui avaient quitté de manière plus ou moins forcée ou spontanée les différents pays. Fondamentalement de nature économique, l'action des coopératives a toutefois privilégié les productions intéressant directement l'Etat - les cultures d'exportation, pourvoyeuses de devises et de taxes -, même si les paysans ont su détourner sur d'autres productions les moyens mis à disposition pour les productions d'exportation (la mécanisation de la culture de l'arachide a aussi profité aux cultures vivrières dans le cas du Sénégal, par exemple, $c f$. Yung, 1992)

Les conditions de leur insertion dans les systèmes socio-politiques locaux et nationaux, la non-prise en compte des contraintes économiques

(3) Cette réaction de défiance ne constitue pas une spécificité afri caine. On la retrouve dans des pays comme le Vietnam, où cette forme d'organisation s'est trouvée instrumentalisée dans des dispositifs de contrôle étroit de la paysannerie par le système politioo-administratif. réelles (faiblesse des dotations initiales entraînant des tensions et des crises) et de nombreux détournements monétaires qui ont caractérisé leur fonctionnement réel ont débouché progressivement sur leur asphyxie, puis sur leur faillite économique. Les producteurs conservent de cette période et de ces expériences une suspicion légitime vis-à-vis de cette forme d'action collective ${ }^{(3)}$. 


\section{Les années 90: des configurations originales et profondément évolutives}

Les dynamiques actuelles résultent des réactions des organisations face aux grands changements intervenus depuis vingt ans: le désengagement des Etats et la privatisation des fonctions économiques, les processus de démocratisation, la libéralisation des échanges internationaux et le poids croissant des bailleurs de fonds dans la définition des politiques nationales. C'est au carrefour des interactions entre dynamiques politiques, économiques, sociales que naissent les configurations spécifiques de formes d'organisation que l'on peut observer aujourd'hui.

\section{Les facteurs qui ont influencé les évolutions des organisations dans la dernière décennie}

\section{Le désengagement des Etats}

Les réformes économiques imposées par les institutions de Bretton-Woods trouvent leur origine dans les crises financières des années 80. Les plans d'ajustement structurel impulsés par le FMI visent à rétablir les équilibres macro-économiques. Dans le secteur agricole, ces réformes élimineront d'abord les soutiens directs à la production (subventions aux intrants) et le retrait de l'Etat des fonctions économiques entraîne la privatisation des entreprises publiques dans les domaines de l'approvisionnement en intrants, de la commercialisation et de la transformation des produits agricoles. Ces changements prennent des visages très contrastés selon les pays et au sein d'un même pays en fonction des dispositifs institutionnels de développement à l'œuvre.

Au Cameroun, par exemple, la société cotonnière parapublique (Sodecoton), dont la privatisation est prévue de longue date, a réussi à préserver le caractère intégré de la filière et assure la mise en œuvre de projets sur financements externes. En revanche, la suppression de la Sodecao a conduit les producteurs de café et de cacao à se confronter directement au marché tant en ce qui concerne les approvisionnements en intrants qu'en ce qui concerne la commercialisation. Des regroupements fédératifs s'opèrent dans les organisations liées au cacao, qui jouent un rôle croissant dans la mise en œuvre de services aux producteurs et qui intègrent progressivement l'interprofession café-cacao. Au Bénin, au contraire, le poids de la filière coton dans l'économie nationale induit une polarisation des organisations à l'origine créées par les structures publiques et parapubliques. Ce sont en effet les fonctions liées à la filière coton (gestion des approvisionnements, commercialisation primaire) - assurées jusque-là par les services publics - qui sont transférées aux groupements villageois regroupés en unions sous-préfectorales depuis 1991. Ensuite, ces organisations prendront l'initiative de constituer en 1995 des unions départementales, puis une fédération nationale (Fupro Bénin), affirmant ainsi leur autonomie. 
(4) La Fédération des planteurs de café de Guinée couvre essentiel. lenent les préfectures de la région forestière, la Fédération des paysans du Fouta-Djallon regroupe les producteurs de moyenne Guinée, la Fédération des organisations paysannes de basse Guinée concerne les zones littorales et la Fédération des producteurs de coton regroupe les producteurs de coton de haute Guinée.
La Guinée présente une situation spécifique sur le plan politique et économique du fait de la rupture politique par rapport à la France lors de l'indépendance. Lors de l'avènement de la seconde république, en 1985, l'Etat se retire des activités productives et, dans le secteur agricole, adopte une posture pragmatique à travers une politique de projets agricoles chargés de mettre en place des organisations de producteurs à vocation technicoéconomique selon les spécificités de chaque région. A ce jour, quatre fédérations régionales traduisant des dominantes régionales en matière de production agricole sont opérationnelles ${ }^{(4)}$. En mai 2000, on observe la création du Conseil national des organisations paysannes de Guinée, qui regroupe ces fédérations sur des enjeux communs.

\section{La privatisation des fonctions économiques}

Les processus de privatisation sont indissociables de la dynamique de désengagement de l'Etat. Ils revêtent cependant pour les organisations paysannes une importance plus ou moins forte selon l'influence des facteurs suivants dans les situations où elles se trouvent: un marché national plus ou moins protégé, des filières administrées en cours de libéralisation et leur articulation plus ou moins directe sur le marché mondial.

Les associations villageoises engagées dans la filière riz au Mali (zone de l'Office du Niger) bénéficient des acquis techniques et économiques des réformes engagées depuis maintenant plus de quinze ans avec la réhabilitation des infrastructures hydro-agricoles et la mise au point d'un modèle technique intensif performant (Jamin et al., 1990). Les producteurs jouissent d'un certain degré de protection vis-à-vis des importations, mais leurs organisations se trouvent confrontées au marché et à ses acteurs locaux tant en ce qui concerne l'approvisionnement en intrants (et le crédit) qu'en ce qui concerne la commercialisation (Kuper et al., 2002). Nombre d'entre elles se trouvent en situation difficile vis-à-vis des banques; le manque de règles et de recours juridiques rend difficile leur émergence en tant qu'acteurs économiques à part entière, malgré la mise en place de centres de prestation de services pour le renforcement des capacités en gestion, l'amélioration des contrats avec les commerçants et des recours juridiques en cas de litige commercial.

Face à ces dysfonctionnements, des organisations syndicales voient le jour, en particulier le Syndicat des exploitants agricoles de l'Office du Niger (Sexagon), structure la plus représentative aujourd'hui pour négocier avec les partenaires économiques et financiers ainsi qu'avec les structures de l'Etat.

En Guinée, les situations sont fortement contrastées selon les filières et leur environnement économique. La Fédération des paysans du Fouta-Djallon (FPFD), sur les plateaux et notamment dans les zones irriguées des basfonds a développé un système intensif pour la production maraîchère (pomme de terre, oignon et tomate). Organisation à dominante technicoéconomique fortement structurée autour de ces filières, elle commercialise ses produits à travers des contrats passés avec les commerçants de la place 
pour approvisionner Conakry, notamment. La concurrence avec les produits importés d'Europe à certaines périodes a conduit la FPFD à engager des négociations avec les pouvoirs publics afin d'aboutir à une régulation de ces importations et de permettre ainsi la commercialisation des produits locaux. En ce qui concerne la Fédération des planteurs de café de Guinée (FNPCG) et son insertion commerciale, la situation est plus complexe $\mathrm{du}$ fait de la combinaison de plusieurs facteurs: la faiblesse de la monnaie guinéenne, le jeu des commerçants intervenant sur la filière pour lesquels le café n'est qu'un moyen d'accéder à des devises et les évolutions erratiques et in fine baissières du marché mondial du café. Face aux difficultés récurrentes, la FNPCG a revu sa stratégie en la matière et fait le choix de se concentrer sur des fonctions d'appui à la mise en place de filières de qualités différenciées sans intervenir directement dans le processus commercial (Berthomé et al., 1999).

\section{Les processus de démocratisation}

Les réformes politiques engagées à la suite des réformes économiques conduisent à des processus de démocratisation d'ampleur et de profondeur variables selon la nature des régimes politiques en place dans les différents pays. Même si dans certains pays cette ouverture démocratique reste fragile et limitée (Tchad, Togo) ou demeure encore une utopie non prioritaire pour des situations de conflit armé ou de post-conflit (Sierra Leone, GuinéeBissau), les conséquences des changements politiques sur les dynamiques d'organisation sont essentielles et il existe une liaison directe entre ouverture démocratique et dynamiques d'organisation du milieu rural.

Au Sénégal, le processus de démocratisation est ancien; trois partis politiques sont reconnus dès 1976 et le multipartisme est instauré en 1981. Au niveau des organisations, des statuts juridiques suffisamment souples (ONG et GIE) permettent l'émergence d'associations de développement multisectorielles qui vont se structurer progressivement en fédérations régionales, puis au niveau national au sein de la Fédération des ONG du Sénégal (FONGS) dans les années 80. Elle jouera un rôle moteur dans le regroupement des principales organisations fédératives nationales au sein du Comité national de concertation des ruraux (CNCR) à partir de 1993. Dans ce mouvement de structuration à l'échelon national, qui conduit à la formation du CNCR, se retrouvent associées, d'une part, les fédérations émancipées de la tutelle de l'Etat (fédérations de coopératives structurées sur la base des grandes filières créées durant la période des coopératives) et, d'autre part, des associations regroupées dans la FONGS qui se sont souvent construites en opposition aux formes directives de l'intervention de l'Etat. La phase de désengagement de l'Etat conduit au renforcement du mouvement associatif et permet une réelle prise de parole, puisque le CNCR peut faire état d'une représentativité significative.

Dans les autres pays, il faudra souvent attendre la mise en œuvre des orientations définies lors du sommet franco-africain de La Baule, qui font écho aux pressions plus ou moins fortes des institutions de Bretton-Woods, 
de l'Union européenne et de certaines coopérations bilatérales, pour que s'engagent des discussions sur les conditions d'une ouverture démocratique. Au Bénin, en 1989, la Conférence nationale souveraine inaugure avec succès ce processus en mettant fin à dix-sept ans de régime de parti unique d'inspiration marxiste et en décidant de la tenue d'élections libres en 1990 et 1991. Depuis cette date, d'autres élections se sont déroulées et l'alternance se passe sans heurts notables: le multipartisme est réel, la presse est libre et les divers organes du pouvoir fonctionnent. Cette expérience va constituer une référence pour tous les autres pays de la sous-région, mais ne sera jamais égalée. Au Cameroun, si la démocratisation ne s’accompagne pas d'un changement des tenants du pouvoir, elle n'en est pas moins une réalité dans ses formes, puisque l'ouverture politique instaure le multipartisme formel en 1990, entrainant la création d'une multitude de partis politiques, et libère la presse de la tutelle étatique.

Le Syndicat des cotonniers et des vivriers (Sycov) au Mali naît dans la zone cotonnière en 1991 dans l'espace politique ouvert par le " vent démocratique » qui accompagne la chute du régime Traore. Ce syndicat entretient depuis lors des relations difficiles avec la société cotonnière (CMDT). L'absence de partenariat véritable, la stratégie délibérée d'affaiblissement du syndicat de la part de la CMDT et la passivité de la plupart des partenaires au développement conduisent à une crise majeure de la filière coton en 2001 (effondrement de la production suite à une grève des semis).

En revanche, les dynamiques de ce type sont beaucoup plus prudentes dans l'ouest du Burkina Faso (zone cotonnière) en raison de la nature encore autoritaire du pouvoir politique. On assiste cependant à l'émergence de l'Union nationale des producteurs de coton (UNPCB) qui est amenée à cogérer la filière avec la société cotonnière.

Le mouvement n'est cependant pas univoque, comme en témoigne le cas de la Guinée, où des organisations paysannes structurées jusquau niveau national ont pu voir le jour alors que le processus de démocratisation politique tarde à se mettre en œuvre. On se trouve dans une situation où le désengagement de l'Etat des fonctions économiques se traduit par la reconnaissance de fait d'organisations à vocation technico-économique sans que leur soit pour l'instant reconnu un statut officiel.

\section{Spécificités des configurations actuelles}

Les configurations actuelles résultent des interactions entre les dynamiques internes propres aux organisations et les changements survenus dans leur environnement économique et institutionnel, notamment durant la dernière décennie.

Le désengagement de l'Etat - en homogénéisant le contexte dans lequel évoluent les organisations en le rendant à la fois plus difficile pour les producteurs et plus ouvert aux initiatives de leurs organisations, en autonomisant des organisations jusque-là sous tutelle - favorise le rapprochement entre des types d'organisations différents et la prise de conscience de l'existence d'intérêts communs. 
Les organisations paysannes dans leurs formes actuelles, les fonctions qu'elles assurent et le rôle qu'elles jouent en matière de développement agricole et rural ne répondent pas à la mise en œuvre de modèles préétablis comme dans la période qui a suivi les indépendances (Bosc et al., 2001).

\section{Les organisations actuelles apparaissent comme des formes hybrides qui produisent des biens de natures diverses}

Elles font clairement partie du secteur privé, mais elles sont des formes hybrides qui développent de multiples fonctions qui ne sont pas toutes orientées vers la recherche du profit. A côté des fonctions économiques dont certaines - mais pas toutes - peuvent faire l'objet de rémunération, elles tendent à assurer des fonctions de représentation et de défense des intérêts, des fonctions sociales et des fonctions de coordination. Ces fonctions se traduisent par la production de biens de natures différentes: des biens privés utilisés individuellement par leurs adhérents dans le cadre de leurs activités de production, des biens collectifs pour l'organisation ou pour l'ensemble des membres de l'organisation jusqu'en allant à des biens qui ont les caractéristiques de biens publics notamment en matière d'alphabétisation, de formation, d'amélioration des pratiques culturales pour une durabilité des systèmes de production ou de services sociaux.

\section{Les fonctions assumées et leurs relations avec les autres acteurs évoluent en fonction des changements du contexte}

Les formes d'organisation actuelles sont des formes transitoires: il ne semble pas exister de déterminisme ou de règle qui permette de définir a priori les évolutions possibles ou les choix les plus pertinents en matière d'organisation. C'est de l'interaction avec son environnement et des choix effectués en fonction des évolutions de cet environnement que résultent les champs d'investissement d'une organisation donnée dans un contexte donné. Une organisation peut être amenée à remplir de multiples fonctions afin de répondre aux défaillances de marché dans d'autres secteurs que le secteur agricole. C'est notamment souvent le cas des services sociaux, par exemple; mais la prise en charge de ce type de fonction n'est assurée qu'en l'absence d'autres structures spécialisées. Contrairement à la période suivant immédiatement les indépendances, il n'est pas fait référence à un modèle d'organisation défini a priori, comme cela fut le cas avec les coopératives. Dans certains cas, relativement rares, les formes coopératives connaissent des succès bien réels, comme dans le cas de la Fédération Kafo Jiginew, créée en 1988, qui regroupe des caisses mutuelles d'épargne et de crédit dans la zone de Mali-Sud. Pour éviter le rappel d'expériences antérieures, le terme " coopérative » a cependant été proscrit lors du lancement.

\section{Des dynamiques fédératives qui traduisent le souci de participer aux débats de politique agricole}

La dernière décennie a vu de manière massive en Afrique (mais aussi dans d'autres situations) l'émergence de mouvements fédératifs aux échelons 
régionaux et nationaux, avec comme perspective la défense des intérêts des producteurs et la prise de parole dans les débats de politique agricole et rurale. Le CNCR au Sénégal en est une parfaite illustration, tout comme les mouvements fédératifs plus récents qui se mettent en place au Burkina Faso et au Cameroun. Par ailleurs, à l'échelon ouest-africain à travers le Réseau ouest-africain des organisations paysannes et des producteurs agricoles de l'Afrique de l'Ouest (Roppa), des synergies tendent à se développer qui se focalisent, d'une part, sur la question de la participation des organisations paysannes aux discussions en matière de politiques agricoles et, d'autre part, sur la mise en commun de moyens pour le renforcement des capacités des organisations. Ces dynamiques fédératives se retrouvent dans les deux mouvements internationaux qui regroupent les organisations de niveau national qui se partagent actuellement la représentation et la prise de parole des organisations paysannes à l'échelle intercontinentale la Fédération internationale des producteurs agricoles (Fipa), créée à la fin de la Seconde Guerre mondiale, et Via Campesina, créée plus récemment.

Des initiatives collectives telles que le Réseau APM Afrique (Agricultures paysannes et modernisation) permettent par ailleurs de fédérer des réflexions de responsables d'organisations, de chercheurs et de praticiens de l'appui aux organisations, avec l'objectif clair de faire émerger des services aux organisations contrôlées par ces dernières. Une initiative récente de ce réseau concerne la mise en œuvre d'un cycle de formation par alternance pour de jeunes responsables paysans africains (Université paysanne africaine) qui a comme finalité d'éclairer les mécanismes de changement économique, politique et institutionnel à l'œuvre dans le secteur agricole et d'engager une réflexion stratégique au sein des organisations afin de les amener à défnir leurs propres positionnement et projets stratégiques.

Les organisations paysannes et rurales opèrent ainsi aux différents niveaux de structuration, depuis le niveau local jusqu'au niveau international. Elles présentent toutefois la spécificité d'être ancrées au niveau local, là où se situe la demande effective de services de la part de leurs adhérents. C'est bien en répondant aux demandes concrètes de leurs membres que les organisations construisent leur légitimité; c'est aussi à ce niveau que peut se développer une nouvelle vision du " politique " à travers les formes de gestion qui sont mises en œuvre. Elles participent ainsi à la construction de liens entre les champs de l'économique et du social, d'une part, et de l'économique et du politique, d'autre part, et ce aux différentes échelles de gestion issues du processus de décentralisation.

\section{Conclusion générale}

Ces organisations agricoles et rurales africaines en profonde recomposition, qui connaissent toutes des processus d'ajustement et d'adaptation permanents, traduisent des tentatives inégales et souvent incertaines d'adaptation 
à des environnements économiques et institutionnels en profonde mutation. La principale question de la période à laquelle elles sont confrontées est celle de l'avenir des agricultures familiales, dont le développement sous protection des Etats a été une caractéristique majeure de la croissance agricole au cours du XXe siècle.

L'oligopole d'Etats-nations qui gérait les offres nationales sur les marchés internationaux a été démantelé par le processus de libéralisation. Il tend à être remplacé de plus en plus, avec de nombreuses résistances, par un oligopole de firmes qui intègrent de façon progressive l'ensemble des filières de production, transformation et commercialisation, allant parfois jusqu'au contrôle de la production par différentes formes de contractualisation (Daviron et Gibbon, 2002). Ce processus d'intégration par les firmes s'accompagne d'un phénomène de segmentation de plus en plus marqué des agricultures qui voit une opposition croissante entre une petite frange d'agriculture d'entreprise et une grande masse d'agriculteurs poussés progressivement vers la marginalisation économique et sociale (Losch, 2002). Il est accentué dans les pays dits en développement par les politiques promues par les agences internationales.

Ce mouvement, qui fait écho au credo libéral - avec promotion du secteur privé d'un côté et gestion de la pauvreté de l'autre -, connaît une amplitude mondiale. Il met non seulement en jeu la forme et la nature de l'organisation de l'agriculture, mais aussi, plus globalement, les projets de société (Mercoiret et al., 2000) et la capacité des groupes sociaux à porter et défendre leurs projets collectifs.

Il s'agit là d'un défi majeur pour l'ensemble des organisations de producteurs agricoles et ce défi est assurément accentué dans les situations africaines, où les caractéristiques des configurations nationales et leur insertion internationale créent des handicaps durables. En Afrique aussi, les marges de manœuvre ne pourront être acquises que par le renforcement des capacités d'action et de négociation permettant un rapport de force moins défavorable. Cette évolution nécessaire, qui appelle un effort majeur en termes d'information et de formation, mais aussi d'amélioration de l'environnement institutionnel par la construction de politiques publiques adaptées, dépasse le strict cadre de la " coopération » agricole. Et les organisations paysannes devront dépasser un registre de débat limité à la production agricole pour rechercher des alliances avec d'autres secteurs et acteurs de la société. 


\section{Bibliographie}

Augé M., 1973, «L'illusion villageoise, limites sociologiques et politiques du "développement" villageois en Côte d'Ivoire ", Archives internationales de sociologie de la coopération et du développement, 34, pp. 240-251.

Banque mondiale, 1989, La Banque mondiale et le Sénégal, 1960-1987, Washington, USA, Banque mondiale, Département de l'évaluation rétrospective des opérations, rapport $\mathrm{n}^{\circ} 8041$, 185 pages.

Berthomé J., 1990, «Les associations villageoises de développement en Afrique de l'Ouest ", Economie et humanisme, 314, pp. 15-27.

Berthomé J., Bosc P.-M., Dardé C., Mercoiret J., 1999, Etude de capitalisation sur les dynamiques d'organisation paysanne en Guinée, vol. 1: Rapport général, vol. 2: Annexes, vol. 3: Support pédagogique, Montpellier, France, Cirad-Tera, 250 pages. Bosc P.-M., Eychenne D., Hussein K., Losch B., Mercoiret M.-R., Mackintosh-Walker S., 2001, "The role of rural producer organisations (RPOs) in the world bank rural development strategy ", Reaching the rural poor, Cirad-Tera, ODI, 161 pages.

Campagne P., 1998, "Globalisation, systèmes agroalimentaires et paysanneries ", in Symposium "Globalisation et systèmes agroalimentaires", Caracas, 15-17 juillet 1998, 24 pages.

Chauveau J.-P., 1994, " Participation paysanne et populisme bureaucratique, essai d'histoire et de sociologie de la culture du développement ", in Jacob J.-P., Lavigne Delville Ph. (dir.), Les associations paysannes en Afrique, organisations et dynamiques, Karthala, pp. 25-60.

Colin J.-P., Losch B., 1990, "Touche pas à mon planteur, réflexions sur les "encadrements" paysans à partir de quelques exemples ivoiriens ", Politique africaine, 40, pp. 83-99.

Daviron B., Gibbon P., 2002, "Global commodity chains and African export agriculture", Journal of agrarian change, vol. 2, $\mathrm{n}^{\circ} 2,137-161$.
Gentil D., 1988, "Organisations coopératives et Etat, Afrique francophone (1960-1985)", Mondes en développement, 61 (16), pp. 113-126. Gentil D., Mercoiret M.-R., 1991, "Y a-t-il un mouvement paysan en Afrique noire? " Revue Tiers-Monde, 32 (128), pp. 867-886.

Hervieu B., Guyomard H., Bureau J-C., 2000, "L'avenir des politiques agricoles », in Ifpri, Rapport Ramses 2001, Dunod, Paris, pp. 115-131. Jamin J.-Y., Zerbo A. D., Berete O, Sanogo M. K., Keita M., 1990, "Conditions de l'intensification de la culture irriguée dans les grands périmètres sahéliens: l'expérience du projet Retail à l'Office du Niger ", Cahiers de la recherchedéveloppement, $\mathrm{n}^{\circ} 27$, pp. 47-60.

Kuper M., Tonneau J.-P., Bonneval P (édit.), 2002, L'Office du Niger, grenier à riz du Mali: succès économiques, transitions culturelles et politiques de développement, Montpellier, Cirad. Losch B., 2000, "Coup de cacao en Côte d'Ivoire", Critique internationale, n 9, pp. 6-14. Losch B., 2001, Identification d'un programme de formation pour les responsables professionnels agricoles du secteur café-cacao en Côte d'Ivoire, Cirad, APM Afrique, novembre 2001, CiradTera, $n^{\circ} 68 / 01,15$ pages et annexes.

Losch B., 2002, "La multifonctionnalité face aux défis des agricultures des Suds: une perspective de refondation des politiques publiques ", Colloque de la Sfer, "La multifonctionnalité de l'activité agricole et sa reconnaissance par les politiques publiques ", Paris, 21-22 mars 2002, 23 pages.

Mc Michael P., 1996, Development and social change, a global perspective, Pine, Forge Press, Thousand Oaks, London, New Delhi, 1996.

Mercoiret M.-R., Vuarin P., Berthomé J., Gentil D., Bosc P.-M., 1997, Etats désengagés, paysans engagés, perspectives et nouveaux rôles des organisations paysannes en Afrique et en Amérique latine, compte rendu de l'Atelier international 
de Mèze, France, 20 au 25 mars 1995, Paris, France, FPH, Dossier pour un débat, 189 pages. Mercoiret M.-R., Munoz J. P., Minla Mfou'ou J., Berthomé J., Bosc P.-M., 2000, Les organisations paysannes et indigènes face aux défis de la mondialisation, t. 1: Mise en cuvre et résultats de la première phase, projet fédérateur de recherche-action, Réseau APM Afrique, Cirad, Ciepac, Riad, FPH.

Reboul C., 1987, "Les associations de village de la vallée du fleuve Sénégal ", Revue TiersMonde, 1987, 28 (110), pp. 435-440.

Robinson K. E., 1950, "The "sociétés de prévoyance" in French West Africa ", Journal of African administration, 2, pp. 29-35.
Sarraut-Woods J., 1998, La politique française de coopération en matière de développement rural, des certitudes à la dérive, Observatoire permanent de la coopération française, rapport 1998, Karthala, Paris, pp. 47-94.

Stiglitz, J. E., 2002, La grande désillusion, Fayard, 325 pages.

World Bank, 2001, Attacking poverty, world development report, Washington DC.

Yung J. M., 1992, «Innovation et monétarisation, bassin arachidier, Sénégal ", in Bosc P.M., Dollé V., Garin P., Yung J.-M., Le développement agricole au Sahel, t. III, Cirad, collection " Documents systèmes agraires ", $\mathrm{n}^{\circ} 17$, Montpellier, pp. 1-21. 